\title{
Generation of 3D ellipsoidal laser beams by means of a profiled volume chirped Bragg grating
}

\author{
S. Yu. Mironov ${ }^{*}$, A.K. Poteomkin ${ }^{1}$, E.I. Gacheva ${ }^{1}$, A.V. Andrianov ${ }^{1}$, V.V. Zelenogorskii ${ }^{1}$, R. Vasiliev ${ }^{2}$, \\ V. Smirnov ${ }^{2}$, M. Krasilnikov ${ }^{3}$, F. Stephan ${ }^{3}$ and E.A. Khazanov ${ }^{1}$ \\ ${ }^{1}$ Institute of Applied Physics RAS, Nizhny Novgorod, Russia \\ ${ }^{2}$ OptiGrate Corp, 562 South Econ Circle, Oviedo, Florida 32765-4311, USA \\ ${ }^{3}$ Deutsches Elektronen-Synchrotron a Research Centre, Platanenallee 6, Zeuthen, Germany, D-15738 \\ Sergey.Mironov@mail.ru
}

A method for shaping photocathode laser driver pulses into 3D ellipsoidal form has been proposed and implemented. The key idea of the method is to use a chirped Bragg grating recorded within the ellipsoid volume and absent outside it. If a beam with constant (within the grating reflection band) spectral density and uniform (within the grating aperture) cross-section is incident on such a grating, the reflected beam will be a 3D ellipsoid in space and time. 3D ellipsoidal beams were obtained in experiment for the first time. It is expected that such laser beams will allow reducing the electron bunch emittance when applied at $\mathrm{R}^{ \pm}$photo injectors.

\section{PACS: 42.60.By, 42.60.Jf, 42.30.Va}

\section{Introduction}

One of the most important problems in present-day linear accelerators is generating electron bunches with low emittance. An ultimate solution of this problem lies in obtaining electron bunches with uniform charge density in ellipsoidal volume (the Kapchinskij-Vladimirskij distribution) [1]. Generation of such bunches is a complicated problem that has not been solved so far. The first step towards its solution is generation of 3D ellipsoidal laser beams and using them for electron photoemission.

A well-known tool to approach the goal is modulation of spatial and spectral parameters of laser beams by means of spatial light modulators (SLM) [2]. A schematic diagram of the optical system used for "cutting out" a profiled 3D beam, ellipsoidal in particular, from a laser beam with 3D Gaussian spatiotemporal intensity distribution is presented in Fig. 1. This scheme is based on an optical compressor/stretcher with zero frequency dispersion. The compressor consists of a pair of identical diffraction gratings and a Kepler telescope with magnification equal to unity. An amplitude-phase modulator that changes the original beam spectrum at different points of the cross-section to the spectrum corresponding to the ellipsoidal beam is placed in the telescope focal plane. This approach is especially useful for pulses with appreciable linear frequency modulation, when the temporal intensity distribution repeats the spectral intensity distribution [3] and spectrum shaping is equivalent to temporal (longitudinal) shaping.

For simultaneous control of temporal and spatial (transverse) parameters of radiation the telescope should have cylindrical lenses and a 2D modulator (Fig. 1). A spectral mask $M(\omega, y)$ allows shaping the intensity distribution along one transversal coordinate (Y-coordinate in Fig. 1). By rotating a beam coming from the shaper by 90 degrees and organizing the second pass through the shaper one can form a 
symmetric beam with respect to two orthogonal axes. Each transversal cross-section of such a beam will be square, with the square side changing along the Z-axis by ellipsoidal law. Such quasi-3D ellipsoidal beams have been obtained in [2]. The beam may be turned by an angle of 45 degrees, then after quadruple passage through the shaper a structure with octagonal cross-sections close to an axially symmetric one will be formed. It is rather difficult to adjust such a 4-pass shaper, and it has very low efficiency because of a large number of optical components. Therefore, it is not promising for practical applications.

Consequently, axially symmetric distributions cannot be obtained by 3D beam shaping using SLMs. Therefore, in the presented research we proposed and implemented a technique of forming axially symmetric 3D ellipsoidal beams by means of volume chirped Bragg gratings (CBG) with diffraction efficiency profiled over the volume. In contrast to SLMs, these gratings do not demand spatial frequency splitting along the $\mathrm{X}$-axis (like in Fig. 1). Therefore, profiling of the CBG reflection coefficient along two transverse coordinates leads directly to the corresponding beam shaping along two transverse coordinates. Section 2 is devoted to the device itself and the parameters of a profiled CBG. The scheme of the experiment is described in detail in Sec. 3, and the results of measuring the shape of the obtained 3D ellipsoidal beam are presented in Sec. 4.

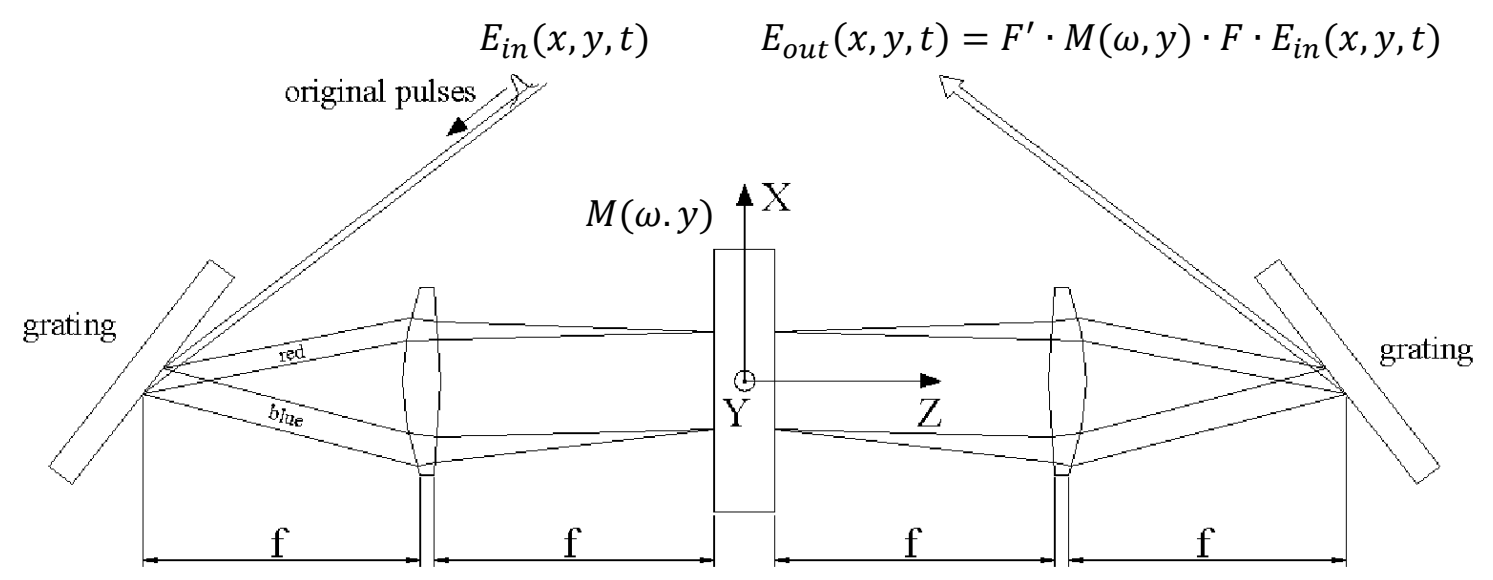

Fig. 1. Schematic diagram of a shaper based on zero dispersion compressor; $F$ and $F^{\prime}$ are operators of forward and inverse Fourier transform, $\mathrm{M}(\omega, \mathrm{y})$ - spectral-spatial mask.

\section{3D Chirped Bragg Grating}

Volume Bragg gratings, including CBG in photothermorefractive (PTR) glass [4], are widely used in different lasers thanks to their high diffraction efficiency, high damage threshold, and temperature and optical stability (see, e.g., [5-7] and references therein). The refractive index modulation period linearly changes along the direction of light propagation. In this case, different wavelengths are reflected from the grating at different depths, thereby introducing linear frequency modulation in the reflected beam.

For 3D ellipsoidal beam shaping we fabricated spatially profiled CBG. In such a grating, the region of transverse reflection for each wavelength is a circle with uniform reflection coefficient. The diameter of the reflection region changes depending on wavelength (longitudinal coordinate of the grating). At the boundaries of the reflection spectral band of the Bragg grating, the transverse size of the reflection region is minimal and for the central reflection wavelength it is maximal. Such a grating allows controlling the reflected beam diameter as a function of longitudinal coordinate. The coordinate is proportional to time and wavelength. We will call these gratings 3D CBGs. They are made of standard CBGs by removing refractive index modulations from the grating areas where reflection is not needed. Reduction of the refractive index modulation amplitude is done by means of ultraviolet radiation with subsequent thermal 
processing to avoid damage of the produced 3D structure. In the present research we used a reflective 3D CBG produced by OptiGrate with the following parameters: aperture 6x6.3 mm, maximum ellipsoid diameter $6 \mathrm{~mm}$, length $35 \mathrm{~mm}$, central wavelength $1029.9 \mathrm{~nm}$, FWHM spectral band $5 \mathrm{~nm}$, and stretching factor $63.3 \mathrm{ps} / \mathrm{nm}$. The reflection coefficient (diffraction efficiency) of the grating was measured as a function of transverse coordinate and wavelength (Fig. 2). Measurements were done by means of a superfluorescent LED. The CBG was placed on an XY linear translator so that the spectral profile of the reflection coefficient could be measured for the entire grating aperture. Reduced efficiency at central wavelength for the position $2 \mathrm{~mm}$ off the aperture center (see Fig. 2a) in comparison with the center position can be explained by finite diameter of the test beam of $\sim 1 \mathrm{~mm}$. The obtained data were used for calculation of the reflection coefficient spatial profile as a function of wavelength (Fig. $2 b$ ). As seen in Fig. $2 b$, the boundary of the reflection coefficient distribution of the profiled Bragg grating is fuzzy along the transverse coordinate. This is explained by finite size of the beams used for erasing the grating and measuring the reflected spectrum.

a)

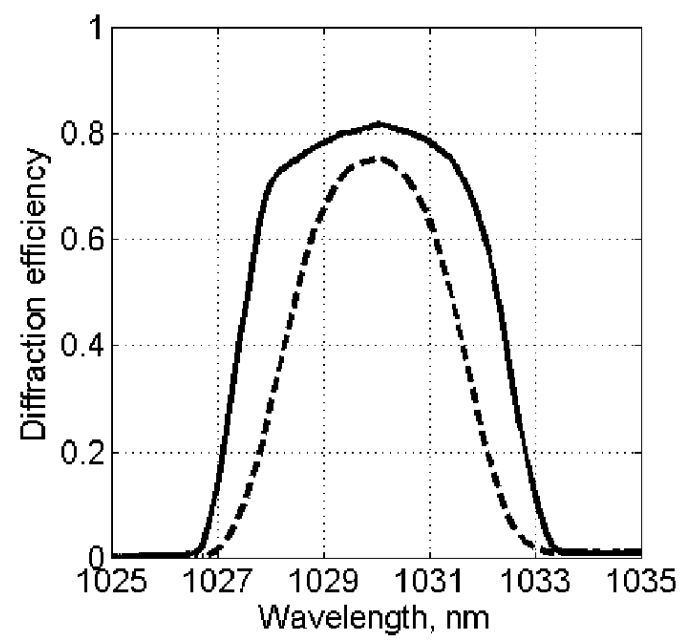

b)

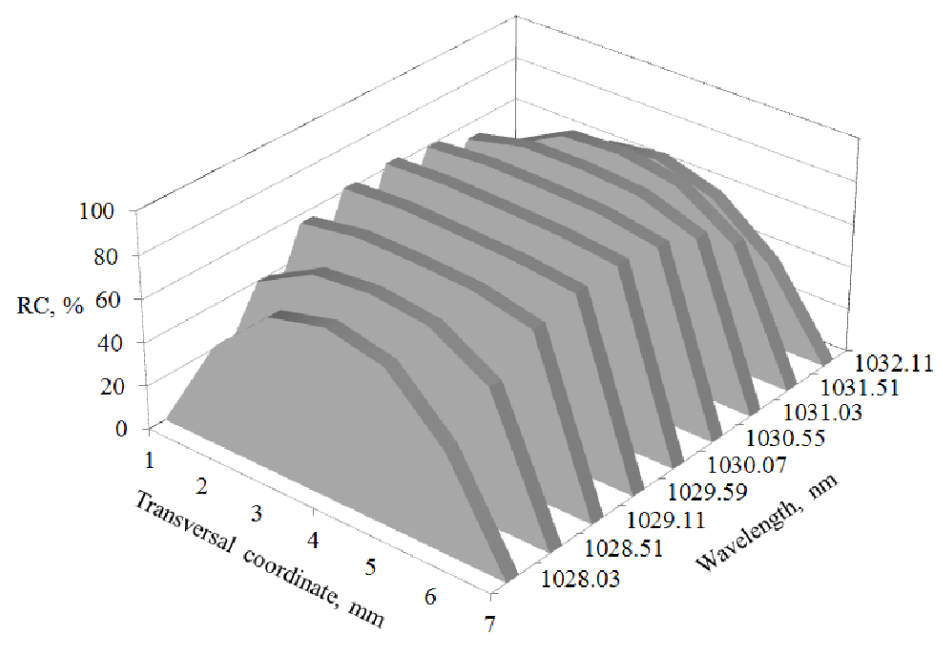

Fig. 2 a). Spectral shape of diffraction efficiency measured in the center (solid line) and $2 \mathrm{~mm}$ off the center (dashed line) of the 3D CBG aperture; b). Grating reflection coefficient as a function of transverse coordinate and wavelength.

If a beam with a diameter over $6 \mathrm{~mm}$, uniform along transverse coordinates, with a rectangular spectrum having a width more than $6 \mathrm{~nm}$ is incident on such a grating, then the reflected beam will have a shape close to an ellipsoid.

\section{Scheme of the experiment}

The schematic diagram of the experimental setup is shown in Fig. 3. A fiber master oscillator 1 based on polarization-maintaining ( $\mathrm{PM}$ ) single-mode $\mathrm{Yb}$ fiber works in the regime of passive mode locking attained by means of SESAM (Semiconductor Saturable Absorber Mirror). The intracavity grating compensates dispersion in the fiber, which provides a pulse duration of $200 \mathrm{fs}$ with spectral width (FWHM) $8 \mathrm{~nm}$, central wavelength $1034.6 \mathrm{~nm}$, pulse repetition rate $47 \mathrm{MHz}$, and average radiation power $0.45 \mathrm{~mW}$. 


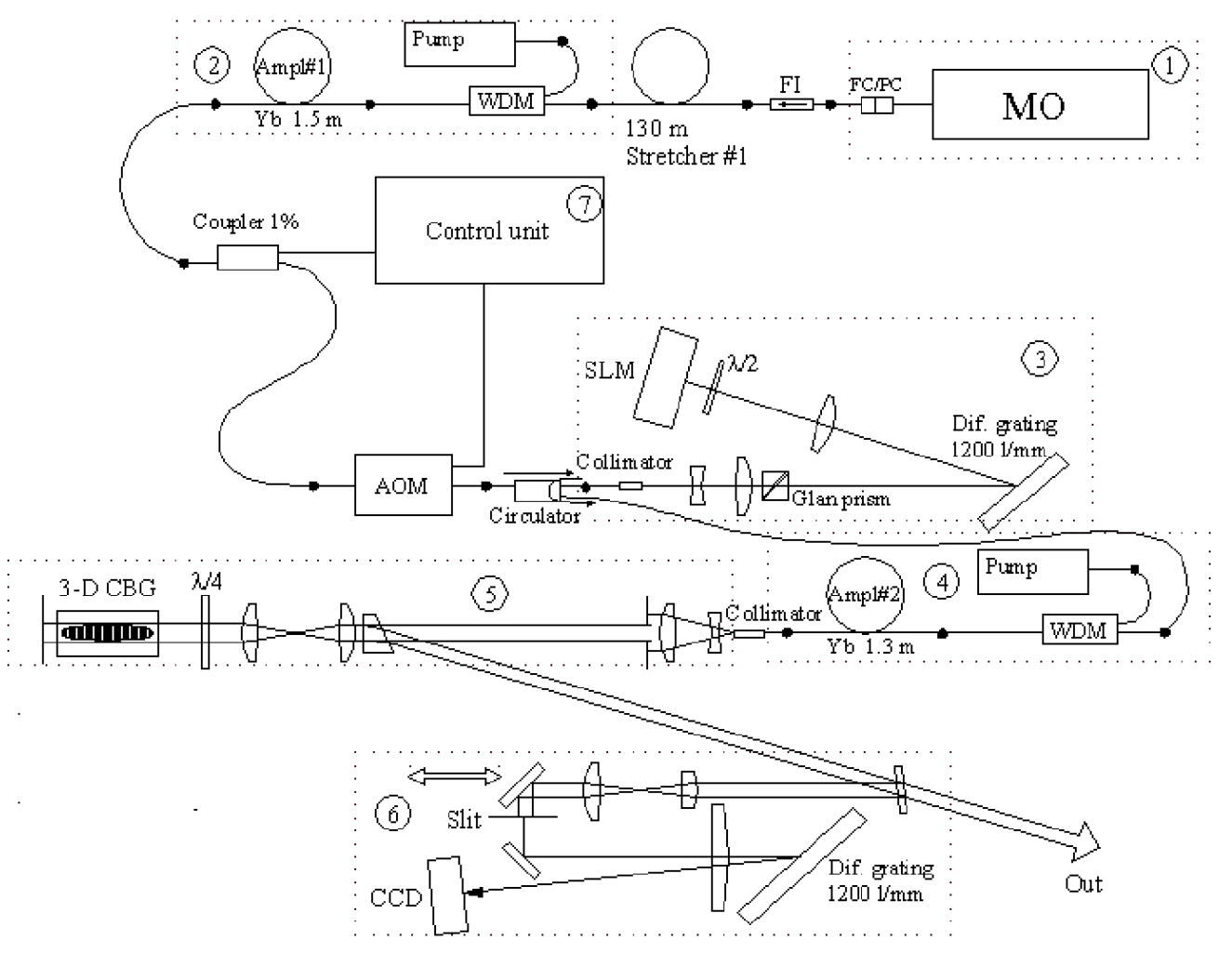

Fig. 3. Schematic diagram of the experimental setup. 1 - fiber master oscillator, 2 - fiber pre-amplifier, 3 spectrum shaper, 4 - fiber amplifier, 5 - shaper based on 3D CBG, 6 - scanning-image spectrograph, 7 - laser control unit.

Pre-amplifier 2 consists of a 1.5 m-long PM-YSF-HI Yb-doped fiber pumped by a laser diode with $400 \mathrm{~mW}$ output power. Between the master oscillator and the amplifier there is a fiber Faraday isolator (FI) and a stretcher, where the pulses are being stretched up to $40 \mathrm{ps}$. The stretcher is made of a $130 \mathrm{~m}$ section of a single-mode PM fiber. A 1\% fiber coupler extracts pulses for controlling the laser operation and synchronizing using an acoustooptical modulator to reduce the pulse repetition rate by a factor of 4 , $8,16,32$ or 64 . It opens for a short time (for about $10 \mathrm{~ns}$ around the transmitted pulse) to reduce the level of luminescence noise.

To control the spectrum of the output radiation a spectrum modulator - spectrum shaper 3 in Fig. 3 was placed between the amplifiers. It is analogous to the shaper in Fig. 1, but with a reflective SLM. As a spherical lens was used in the scheme, only the profile along the spectral coordinate (X in Fig. 1) can be modulated. The radiation reflected from the spectrum shaper 3 was forwarded by a circulator to amplifier 4. In addition to shaping a rectangular spectrum, for effective operation of CBG the spectrum had to be shifted to the region of effective CBG reflection (Fig. 2). Operation of the spectrum shaper is demonstrated in Fig. 4: the solid line corresponds to the spectrum at the input (central wavelength $1032 \mathrm{~nm}$ ), and the dashed line at the output (central wavelength $1029.9 \mathrm{~nm}$ ). 


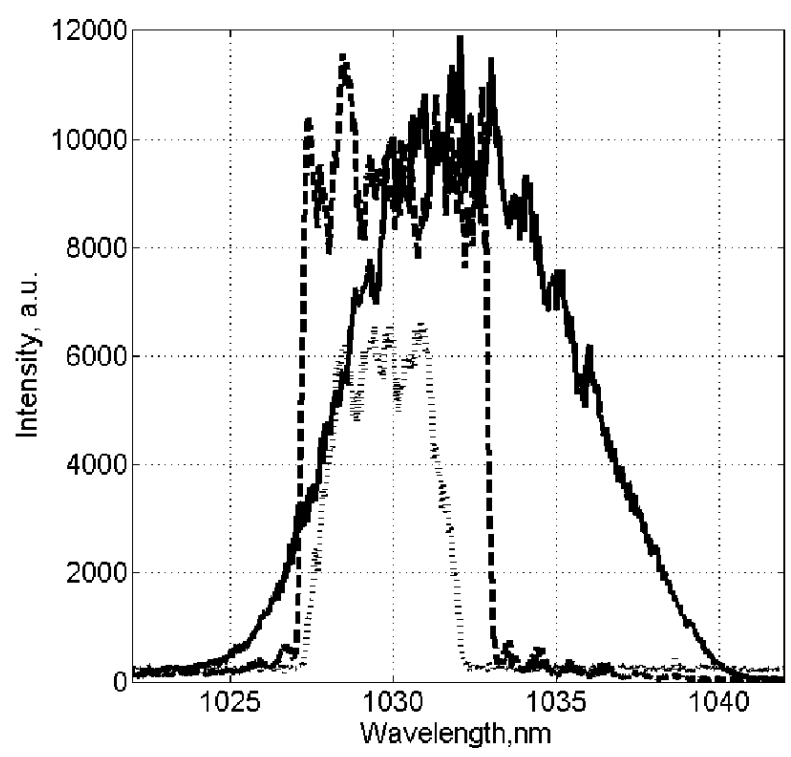

Fig. 4. Radiation spectra before spectrum shaper (solid line), between spectrum shaper and CBG (dashed line), and after CBG on the beam axis (dotted line).

Amplifier 4 differs from pre-amplifier 2 only by the active fiber length of $1.3 \mathrm{~m}$. The average radiation power at the output of amplifier 4 with fully open spectrum shaper is $95 \mathrm{~mW}$. If the shaper cuts a rectangular $5.5 \mathrm{~nm}$ wide spectrum, $3.4 \mathrm{~nm}$ off the center of radiation band (Fig. 4), the efficiency of reflection from the shaper does not exceed several percent. Nevertheless, as amplifier 4 operates in a saturation mode, the radiation output power reduces insignificantly, down to $79 \mathrm{~mW}$. A fiber PM collimator with mode diameter of $1 \mathrm{~mm}$ was spliced to the output of amplifier 4 .

From the description of the 3D CBG given in Sect. 2 it follows that for shaping a 3D ellipsoidal beam, a beam $\sim 5-6 \mathrm{~mm}$ in diameter uniformly filling the aperture and having a rectangular spectrum with center at $1029.9 \mathrm{~nm}$ and width not less than $5 \mathrm{~nm}$ should be forwarded to the grating. The required input radiation spectrum was formed by spectrum shaper 3, which is illustrated in Fig. 4, where the dashed and dotted curves are compared. The transverse intensity distribution was cut by a round diaphragm $4 \mathrm{~mm}$ in diameter from a Gaussian beam $10 \mathrm{~mm}$ in diameter. The image from the diaphragm was transferred to the 3D CBG center by a Kepler telescope. The radiation reflected from the grating was separated from the incident one by a $10^{\circ}$ calcite wedge and a zero-order quarter-wave plate.

\section{3D ellipsoidal beam in experiment}

For 3D diagnostics of the beam shape an image spectrograph 6 (Fig. 3) was used. As was mentioned above, for pulses with appreciable linear frequency modulation, the temporal intensity distribution repeats the spatial intensity distribution [3]. The input slit of the spectrograph was projected through the diffraction grating onto a CCD camera by a lens with a focal distance of $564 \mathrm{~mm}$. The camera recorded 2D spectral intensity distributions. By shifting the beam across the slit we made about $1002 \mathrm{D}$ measurements which were assembled into a 3D intensity distribution of the beam. The spectrograph allows measuring 3D ( $\mathrm{x}, \mathrm{y}$ and lambda) intensity distributions of the shaped pulses. For finding the coefficients for recalculation of $\mathrm{nm}$ to $\mathrm{ps}$, a scanning cross-correlator was used. The nonlinear interaction of the shaped pulses and short Gaussian pulses (compressed to $\sim 200 \mathrm{fs}$ ) coming from another output channel of the fiber part was implemented in a nonlinear crystal to generate the second harmonic signal and measure the cross-correlation function. As the short pulses work as a $\delta$-function in the crosscorrelation function, the generated signal of the second harmonic contains direct information about temporal profile of the shaped pulses. 
The spatial 3D shape of the radiation incident on the CBG is shown in Fig. 5. It is clear from the figure that the shape is close to a cylindrical one. The beam has a diameter of $4 \mathrm{~mm}$ and a duration of $40 \mathrm{ps}$. Note that radiation with such a distribution is of independent significance. The authors of [8] used cylindrical beams for photocathode irradiation and obtained record low values of projected electron bunch emittance for total bunch charges from $20 \mathrm{pC}$ to $2 \mathrm{nC}$.
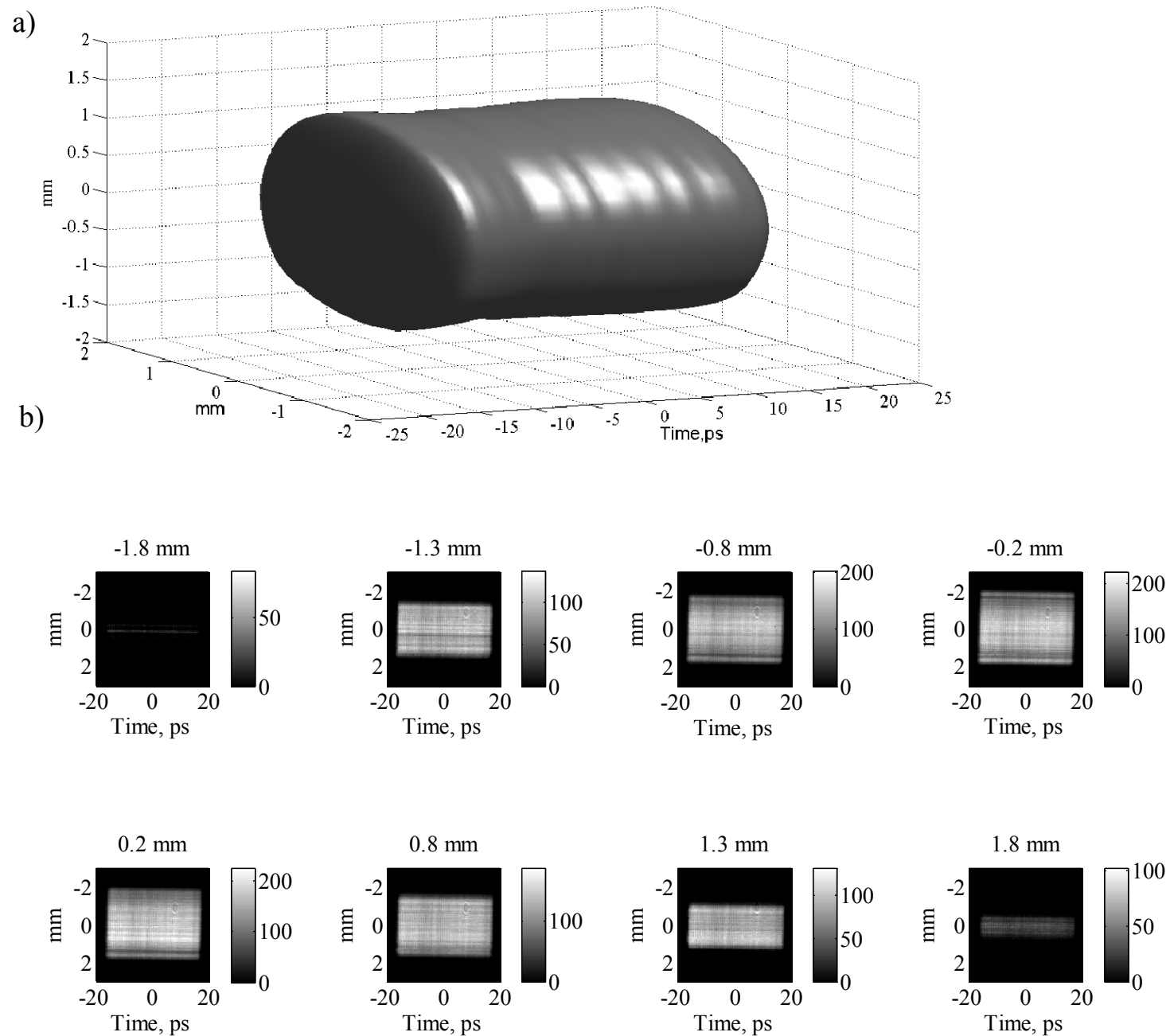

c)
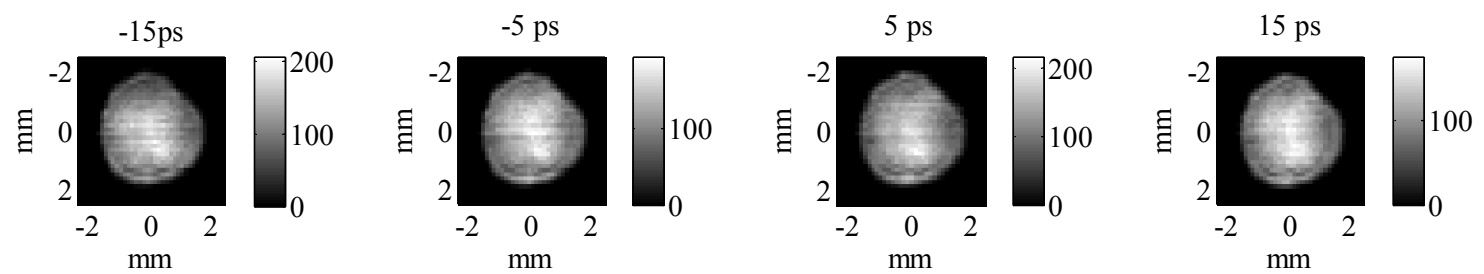

Fig. 5. 3D distribution of the cylindrical beam incident on the CBG (a) and its longitudinal (b) and transverse (c) sections. Longitudinal beam scale -40 ps (the spectrum changes within $1029.9 \pm 2.75 \mathrm{~nm}$ ), transverse scale $-4 \mathrm{~mm}$.

The shape of the beam reflected from the 3D CBG and its 2D sections are presented in Fig. 6: longitudinal sections for different values of X (Fig. 6b), and transverse cross-sections for different values of time (Fig. 6c). The 3D CBG not only shapes an ellipsoidal beam, but also induces an additional frequency chirp. As a result, the duration of the profiled pulse increases up to 264 ps on the beam axis. One can see in Fig. 6 that the transverse sections of the ellipsoidal beam are circular at all moments of time, i.e., the produced shaped laser beam is axially symmetric. This is the key advantage of the proposed approach over the one based on using SLM matrices that ensures only $90^{\circ}$ axial symmetry. 
a)

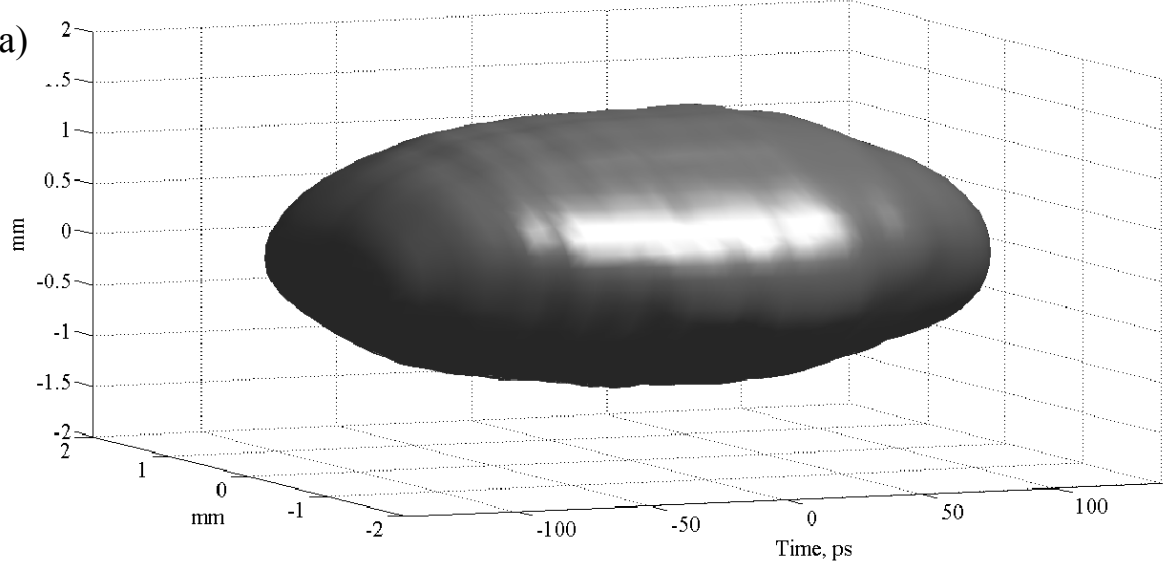

b)
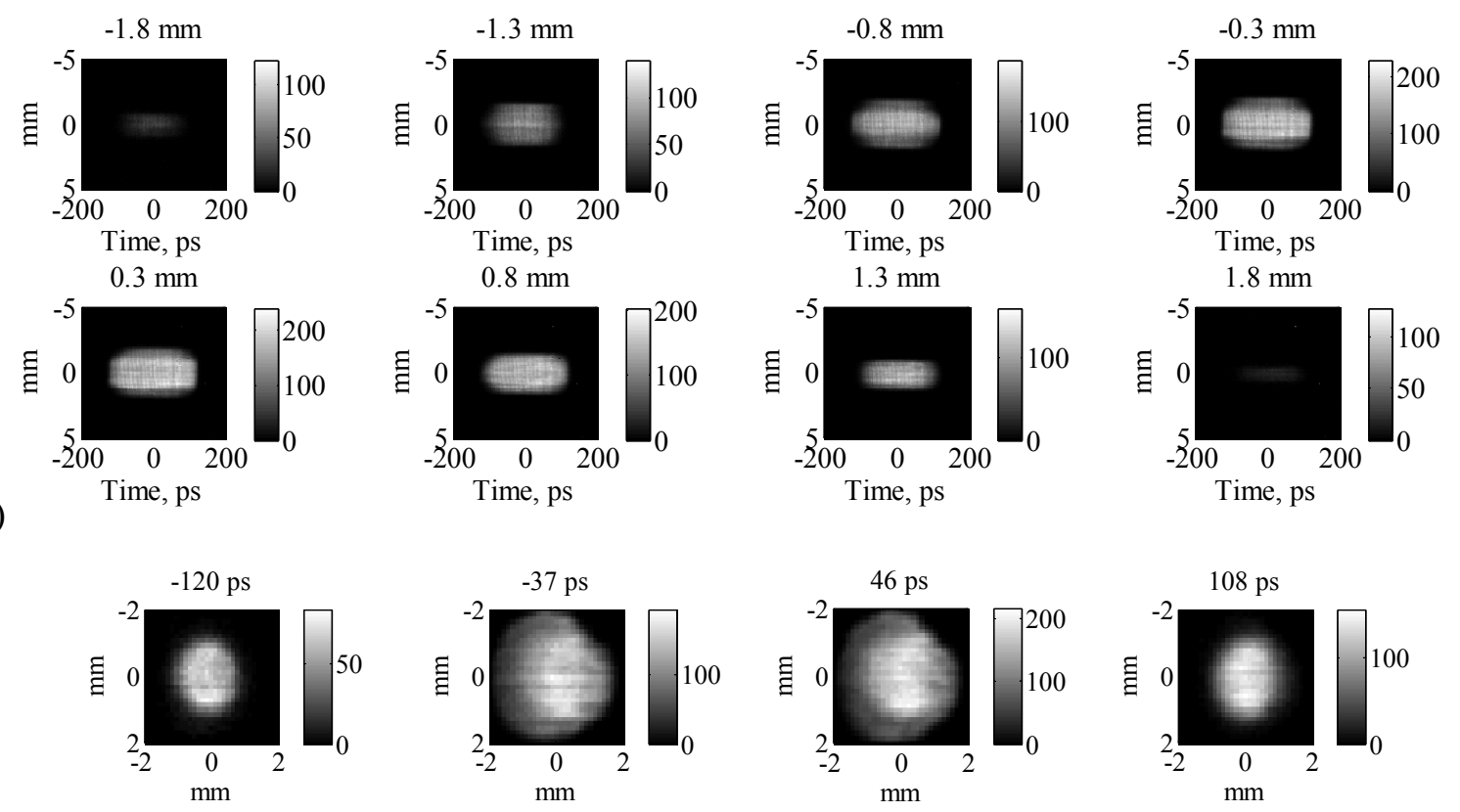

Fig. 6. 3D distribution of the ellipsoidal beam (a), its longitudinal (b) and transverse (c) sections. Longitudinal beam scale $-264 \mathrm{ps}$ (the spectrum changes within $1029.9 \pm 2.5 \mathrm{~nm}$ ), transverse scale of the beam in its widest part $4 \mathrm{~mm}$.

To conclude we emphasize that the formed 3D structure can be scaled in three dimensions. Transverse space scaling of the laser beam is performed by means of a Kepler telescope that changes the beam's transverse size and transfers the image onto the photocathode plane. The features of 3D ellipsoidal beams imaging were described in [9]. Longitudinal (time) scaling may also be accomplished using an additional optical compressor or choosing stretcher and CBG chirps so that the sum chirp corresponds to the needed pulse duration. It is quite an easy task taking into consideration that, when the $\mathrm{CBG}$ is turned by $180^{\circ}$, the sign of its chirp changes to the opposite.

\section{Conclusion}

We have proposed and implemented a basically new approach of shaping 3D ellipsoidal beams for photocathode laser drivers. The key element here is a profiled chirped Bragg grating recorded within an ellipsoid volume and absent outside it. If a beam having a uniform (within the grating aperture) crosssection and constant (within the grating reflection band) spectral density is incident on such a grating, then the reflected beam will be a 3D ellipsoid in space. The basic advantage of this approach is simultaneous beam shaping along two transversal coordinates in space due to grating nonuniformity and 
along the time coordinates due to the frequency-dependent induced delay (chirp). As a result, the quality of the 3D ellipsoidal beams obtained in experiment is greatly improved, which is promising for reducing the emittance of electron bunches injected from a photocathode by such laser beams. Moreover, the proposed optical scheme introduces significantly less losses of energy in comparison with [2], due to using only one pass through the zero-order compressor with SLM without 90 deg beam rotation and additional compensation of energy losses in the fiber amplifier (see fig.3).

\section{Acknowledgement}

This work was supported by the Ministry of Education and Science of the Russian Federation under Contract No.14.Z50.31.0007.

\section{References}

1. I.M. Kapchinskij and V.V. Vladimirskij in: 2-nd Conference on High Energy Accelerators and Instrumentation, p.274-288, CERN, Geneva (1959).

2. S. Yu. Mironov, A. K. Potemkin, E. I. Gacheva, A. V. Andrianov, V. V. Zelenogorskii, M.

Krasilnikov, F. Stephan, and E. A. Khazanov, "Shaping of cylindrical and 3D ellipsoidal beams for electron photoinjector laser drivers," Appl. Opt. 55, 1630-1635 (2016)

3. S.A. Akhmanov, V.A. Vysloukh, and A.S. Chirkin, Optics of Femsotesond Pulses, American Institute of Physics, 1992, New York, $2^{\text {nd }}$ Edition (Nauka, Moscow 1988, in Russian).

4. O.M. Efimov, L.B. Glebov, L.N. Glebova, K.C.Richardson, and V.I.Smirnov, Appl. Opt., 38, 619, (1999)

5. I. Kudryashov and D. Garbuzov, Proc. of SPIE Vol. 6451, 64510P, (2007).

6. M. Smrz, T. Miura, M. Chyla, A. Endo, and T. Mocek: Proc. SPIE 9513, High-Power, High-Energy, and High-Intensity Laser Technology II, 951304 (12 May 2015).

7. M. Hemmer, D. Sánchez, M. Jelínek, V. Smirnov, H. Jelinkova, V. Kubeček, and J. Biegert, Opt. Lett., 40, 451 (2015).

8. M. Krasilnikov, et. al., Phys. Rev. ST Accel. Beams 15, 100701 (2012).

9. Е.И. Гачева, А.К. Потемкин, "Искажения квазиэллипсоидального 3D лазерного импульса-пучка при распространении в свободном пространстве", Известия Вузов Радиофизика, 2015, 58 (4), $304-$ 310. (E.I. Gacheva, A.K. Potemkin, "3D quasi ellipsoidal laser pulse-beam distortions while free-space propagation", Radiophysics and Quantum Electronics, 2015, 58 (4), 277-283.) 